At the same time, Hertz in Germany was making experiments on the way in which electric forces streamed out from a discharging conductor. Using an oscillatory circuit consisting of two widely separated plates joined by a wire, he was able to generate waves as Fitzgerald had predicted. These waves were generated in space and had such energy that they caused little sparks to be emitted by a conductor on which they fell. Hertz showed that Maxwell's theory would account for his radiation in every detail, and he made maps of the lines of force during each phase of the oscillation. These maps were published in NATURE in 1889 , in a translation of Hertz's paper by Sir Oliver Lodge.

Other means of detecting the waves than by the scintillæ they produced were soon developed. Sir Oliver Lodge discovered the principle of the coherer which was afterwards improved by Branly, and in 1894 he showed that Hertz's waves combined with a Branly detector could be used for the sending and receiving of messages in Morse code.

At the same time, Marconi in Italy was studying the application of Hertzian waves to telegraphy, and in 1896 he came to England and was able to interest Sir William Preece, chief engineer to the Post Office, and to secure his co-operation. Marconi persevered with his experiments, and to him must be attributed the great success of wireless telegraphy.

About the same time Sir Oliver Lodge had realised the importance of selectivity and had taken out a patent for a receiver incorporating capacity and selfinduction and capable of being tuned.

The next step, also to be attributed to Marconi, marked the beginning of trans-Atlantic wireless communication. Marconi made use of an elevated aerial connected through a spark gap to earth. The waves generated were consequently in a vertical plane and could travel great distances before becoming attenuated by the earth's resistance. He arranged for a powerful series of signals to be sent out from a station in Cornwall and he himself went to Newfoundland to try to detect them. The experiment was a complete success, and demonstrated that the waves would follow the contour of the earth, a fact which was afterwards explained by the theory of the Heaviside layer. This acts as a kind of whispering gallery and reflects the waves impinging on it.

So far, signalling had been confined to discontinuous trains of waves generated by spark transmitters, but it was realised that there would be a distinct improvement if the signals could be made to consist of modulations in amplitude of a continuous wave of definite frequency, the modulations recurring with a frequency of their own.

The first experimenter to achieve this was Duddell, using an electric arc as part of a circuit containing capacity and inductance. So long as the circuit was not interfered with, the note emitted by the circuit was of uniform tone, but it could be varied by altera. tion of the self-induction or capacity by means of a key. Duddell's arc responded only to low frequency vibrations and was not applicable to the extremely high frequency vibrations needed for effective transmission. Poulsen improved the arc by immersing it in hydrogen under various pressures, and finally obtained an are of really high frequency, which he patented in 1903 .

There still remained the problem of detecting such waves. In 1904, Fleming perceived that the rectifying action of the two electrode valve could be adapted for the detection of ether waves, and he patented the vacuum valve as a detector. At first the valve was only used for the discontinuous system of spark signalling. Later, Fessenden devised the heterodyne system of reception, in which he superimposed on the received wave another of nearly the same frequency, producing low frequency beats which could be detected by the valve and made to operate a telephone. It was now possible to superimpose on a continuous wave the modulation of the human voice, applied by a microphone, and to detect the resulting vibrations at a distant station. This was the beginning of wireless telephony.

The amount of energy received at a station was small and the signals therefore feeble. De Forest conceived the idea of introducing the grid into a valve and supplying the latter with energy from a local high-tension battery. The pulsations of the received wave were applied to the grid, which therefore acted as a regulator, sometimes assisting the flow of current in the valve and sometimes retarding it. The energy of the signals received was supplied by the local battery, the grid merely acting as a relay, putting energy into the disturbance, but not otherwise interfering with it. It was soon found that the three electrode valve could be used for generating continuous waves, at the same time enhancing their power enormously. Thus in 1913 it became feasible to transmit speech and music to great distances.

In 1917 there was further development when the remarkable property of quartz was utilised to control the frequency of transmitted waves. If a crystal of quartz is compressed it becomes polarised, while on the other hand if it is electrified it constricts. This reversible action renders quartz a natural intermediary between mechanical and electrical vibrations. Eccles uses the vibrations of quartz as a transmitter for electrical waves of steady frequency. This frequency is regulated by a tuning-fork adjusted to the frequency desired and operating on a piece of quartz so as to generate waves of the same frequency. After amplification, signals of enormous power can be sent out with a precision of tuning hitherto unequalled. Broadcasting on a large scale was thus made possible. It was initiated in the United States in 1920 and in Great Britain in October 1922.

\title{
Beyond the Milky Way.*
}

THE star-system best known to us is the Galactic system, which is bounded by the Milky Way. About two million such systems of stars can now be observed; they are known as extra-galactic nebulæ. A random collection of extra-galactic nebulæ seems at first to show a bewildering variety of size, shape, brightness, and constitution, but a scientific study soon reduces them to law and order. Rejecting all those which are not seen edge on, we find that by far the greater number of the remainder can be

- Substance of an evening discourse delivered before the British Association, London, on Sept. 29 by Sir James Jeans, F.R.S. arranged in a single continuous sequence, beginning with spheres and ending with flat discs. Since the speed of rotation of a body increases as it shrinks, it seems likely that we may interpret this sequence of nebulæ as one of different stages of development or evolution. If this conjecture is sound, a nebula starting with little rotation at first and shrinking in size would gradually increase its speed of rotation as it shrank, and would move steadily along the sequence as it did so.

The way to test this conjecture is to calculate for ourselves how a mass of rotating gas would change

No. 3231, VoL. 128] 
in shape as it condensed and shrank. Although the mathematical analysis is not simple, and cannot be absolutely precise, it is fairly conclusive ; we find that the evolution of a mass of rotating and shrinking gas would be represented exactly by passage along the sequence.

How did the nebulæ themselves come into being ? The conjecture which at once jumps to the mind is that the nebulæ may have been formed by the same process as the stars; just as the stars came into being as condensations in a tenuous uniformly spread gas - the outer fringes of the nebulæ- so the nebulæ may themselves have previously come into being as condensations in an earlier mass of uniformly spread tenuous gas. This can never be more than a conjecture, but there are strong arguments in its favour.

Differences in size and brightness between nebulæ of the same shape are almost entirely due to a distance effect. This makes it possible to estimate the distances of all nebulæ, even the very faintest, with fair accuracy. The faintest which can be observed photographically in the 100-inch telescope prove to be at the amazing distance of about 140,000,000 light-years. Dr. Hubble finds that the two million nebulæ which lie within this distance are fairly uniformly spaced at about $1,800,000$ light-years apart. We can construct a model, by taking apples and spacing them at about 10 yards apart, until we have filled a sphere a mile in diameter. This will use about 300 tons of apples. This sphere is the part of space we can see in the 100 -inch telescope; each apple is a nebula containing matter enough for the creation of several thousand million stars like our sun: each atom in each apple is as big as Betelgeux, with a diameter equal to, or slightly larger than, that of the earth's orbit.

The circumstance that the nebulæ are fairly uniformly distributed through space certainly supports the conjecture that they may have originated out of a primeval gas spread uniformly through space. Moreover, it can be proved that such a gas would not stay uniformly spread through space but would break up into condensations; and that each condensation would have something like the same mass as the observed nebulæ.

The general breaking-up process of which this is the beginning is in operation throughout the universe. It might be thought that the attractive forces of gravitation would continually draw all the broken pieces together again. The exact reverse appears to be the case. Not only is the substance of the universe for ever being broken into smaller pieces, but these pieces for ever tend to scatter farther and farther apart. Every ray of sunlight that enters our eye carries mass with it ; eight minutes previously this mass was part of the mass of the sun. Every second the sun loses more than four million tons of mass in the form of sunlight and sun-heat. As the result of this continual loss of mass the sun's gravitational hold on its family of planets for ever weakens, and these are driven further off into space. The earth's orbit round the sun is not so much like a circle or ellipse as like a coiled watchspring--a spiral for ever receding into the cold and dark of space.

The same tendency affects the galactic system as a whole. Throughout the universe, all the smaller broken pieces, satellites, planets, stars, are scattering away from one another in apparent opposition to the laws of gravitation.

Still more surprising and sensational is the recent discovery that the largest pieces of the universethe great extra-galactic nebulæ we have been discussing - are to all appearances engaged in a similar scattering. They, too, appear to be running away from us and from one another. Until recently it was thought that on the whole the nearer nebulæ were approaching the galactic system, while the more remote were receding. We now know that the nearer nebulæ appeared to be approaching merely because they happen to lie mostly in the direction towards which the sun is being carried by the rotation of the galaxy; actually we are approaching them. After the sun's motion in the galaxy has been taken into account, all, or nearly all, of the nebulæ appear to be receding from the galaxy. The nearer nebulæ have small speeds, and the more remote nebulæ have greater speeds; in general, speed is approximately proportional to distance. This simple law seems to hold to the very farthest of the nebulæ-Hubble finds that for every million light-years of distance there is a speed of recession of about 105 miles a second. The last nebula to be investigated at Mount Wilson shows a speed of recession of 12,300 miles a second; its distance, as estimated from its faintness, being about 105 million light-years.

On the face of it, this looks as though the whole universe were uniformly expanding, like the surface of a balloon while it is being inflated, with a speed such that it doubles its size every 1400 million years.

There is one strong theoretical argument in favour of regarding the apparent speeds as real. Einstein's original cosmology regarded the universe as being as full of matter as a universe of its size could possibly be without violating the theory of relativity. Recently, Lemaître, of Louvain, has shown that a universe of this type would not be static-there would be an unstable quality about it. The condensation of the primeval gas into distinct nebulæ, and the imprisonment of a large part of the free energy of the universe in these nebulæ, would cause it to start expanding, in which case it would continue to expand, its radius finally increasing exponentially with the time, until it ended up as an empty universe-finite matter spread through infinite space. Throughout the motion the relative speed of recession of any two nebulæ would be exactly proportional to their distance apart, so that, at first glance at least, this theory seems exactly to fit the observed facts. It not only provides a suggestion as to why the nebulæ may be receding. It goes much further, and predicts that they must be receding; if Einstein's relativity cosmology is sound, the nebulæ have no alternative - the properties of the space in which they exist compel them to scatter.

Yet various circumstances suggest a need for caution. For one, the speeds of the nebulæ are not strictly proportional to their distances. Again, the very magnitude of the apparent speeds casts doubt on their reality; they would reduce the whole existence of the universe to a mere flash - at any rate in comparison with what we have recently believed. If they are real, Eddington has calculated that the universe must have started from a radius of about 1200 million light-years, and that its total mass must be about $2 \cdot 3 \times 10^{55} \mathrm{gm}$. which is the mass of $1.4 \times 10^{79}$ protons and an equal number of electrons. So far as we can tell from the masses of the extra-galactic nebulæ, the present average density of matter in space appears to be not less than $10^{-30} \mathrm{gm}$. per c.c., which, with the same amount of matter, would assign a radius of 13,200 million light-years to the universe-only eleven times its initial value. If the motions are real, the universe is only at the beginning of its career; as it appears at present to be doubling in size every 1400 million years, the few doublings which these figures permit cannot have occupied more than 10,000 million years at most.

General calculations on the ages of astronomical bodies point to far longer periods of time than this. The mere act of condensation of the nebulæ was

No. 3231, VoL. 128] 
probably a matter of hundreds of thousands of millions of years. Perhaps there is no real difficulty here; it might well take this long to get the doubling process really going. The real difficulty is that the stars carry intrinsic evidence of having lived through far longer periods than this.

Spectroscopic binaries provide further evidence. Observation reveals a complete sequence; it begins with systems which appear to have just broken into two as the result of rotation-pairs of stars describing circular orbits, and almost in contact - and ends with systems in which the two stars are far apart and describing elliptical orbits. Theory suggests that this observational sequence exactly depicts the evolution of a star which has broken into two as the result of excessive rotation. The outstanding importance of this sequence to our present discussion lies in the fact that the stars at the beginning of the sequence are undoubtedly many times more massive than those at the end. It seems likely that those which are now at the end must have begun at the beginning and lost the greater part of their mass in the form of radiation, and to do this would take millions of millions of years.

Considerations such as these make it very difficult to believe that the universe can be such an ephemeral concern as the apparent speeds of recession of the nebulæ would suggest.

\section{Birthdays and Research Centres.}

Oct. 5, r 86 I.--Sir Thomas Heath, K.C.B., K.C.V.O., F.R.S., honorary fellow of Trinity College, Cambridge, Joint Permanent Secretary to the Treasury, 1913-19, and Comptroller-General of the National Debt Office, 1919-26.

Having published my "Manual of Greek Mathematics" in the early part of this year, I am continuing my life-study of Greek mathematics and astronomy, with the view of keeping pace with the new discoveries in that and allied fields which are continually being made. Thus it is that I was able to include in the "Manual" some interesting things that have come to light since the publication in 1921 of the "History of Greek Mathematics"; for example, an investigation by Archimedes of the problem of inscribing a regular heptagon in a circle (preserved in an Arabic treatise) and some very remarkable results of the study of Babylonian cuneiform inscriptions of 2000-1800 B.C.

I have long wished to write upon the mathematical passages of Aristotle, and hope to proceed with that work if circumstances permit.

Oct. 9, r863.-Prof. A. C. Seward, F.R.S., professor of botany in the University of Cambridge.

Since the publication in 1925 of two papers on Cretaceous plants collected in Greenland by $\mathrm{Mr}$. Holttum and me ten years ago, I have been working through a large consignment of fossils sent to me through the courtesy of colleagues from the museums of Copenhagen and Stockholm. With the help of the additional data thus obtained, I hope to complete in the near future a supplementary account of the rich Cretaceous floras of western Greenland. In this work much assistance has been received from junior coworkers.

Other lines of work are : a more systematic consideration of the bearing of palæobotanical data on the general problem of evolution; and a more critical study of the geographical distribution of plants during the several geological periods.

One of the subjects which I would recommend for treatment is a careful revision of European collections of Tertiary plants.

Oct. I0, I893.-Prof. M. SAfra, F.R.S., University professor of physics, Allahabad.

The Physical Laboratory of the University of Allahabad is one of the oldest and smallest laboratories in India, having been established about thirty years ago, but little research work was done before I took charge in October 1923. Since then, my colleagues and I have been trying our best to organise the laboratory for research work, but I must confess that I am not satisfied with the progress so far made. Research work is being carried on chiefly in spectroscopy and X-rays. At present we are engaged in studying the absorption spectrum of saturated halides of multivalent elements, and on the modification of quanta by photo-ionisation. The work on thermal ionisation has been temporarily suspended owing to lack of space and time. Research students are engaged in the classification of spectral lines. There is a wireless section, with a good broadcasting station, and it is planned to undertake work on propagation of radio in India, but nothing has been achieved, in so far as the co-operation of other universities could not be secured. The staff (total strength, nine) is heavily overworked, as there are about 200 undergraduate and 25 advanced students, and the laboratory is too much congested, for there has been practically no extension since its foundation.

\section{Societies and Academies. LONDON.}

Institute of Metals, Sept. 15* (Annual Autumn Meeting, Zurich).-W. E. Alkins : Experiments in wiredrawing. (2) Notes on the relation between reduction of area by cold-drawing and tensile strength of H.-C. copper. Annealed copper rod 0.435 in. diameter was drawn through straight-sided tapering dies at single drafts of slowly increasing amount. The resulting 'primary' tensile strength reduction of area curve consists of an approximately rectilinear portion up to about 15 per cent reduction and of a smooth curve concave to the reduction axis from 15 per cent upwards. Below 15 per cent, results are consistent with the view that drawing takes place by simple elongation under tensile stress; above $\mathbf{1 5}$ per cent the curve has the form of a rectangular hyperbola, and one asymptote appears to lie suggestively near the limiting tensile strength to which copper can be cold-drawn.-Clement Blazey : Brittleness in copper. The experiments described are a continuation of those described in two other papers already published under the title of "Brittleness in Arsenical-Copper ". It has been found that the brittleness is not restricted to arsenicalcopper, but may be produced in copper free from arsenic.--Edward J. Daniels: The attack on mild steel in hot-galvanising. Experiments have been carried out in the laboratory to determine the rates of attack on mild steel strip when immersed in different brands of zinc at various temperatures between $432^{\circ} \mathrm{C}$. and $540^{\circ} \mathrm{C}$. The influence of the addition of small percentages of aluminium, antimony, and tin has been investigated, and the action of alloys of zinc and cadmium has also been studied.-J. S. Dunn: The oxidation of some copper alloys. The zinc-copper alloys fall, so far as their behaviour under oxidation is concerned, into two classes, fairly sharply separated. Those with copper contents below about 80 per cent all oxidise at essentially the same rate, and all give rise to an oxide which is almost pure zinc oxide.

* Continued from p. 554 .

No. 3231, VoL. 128] 